\section{Primary Pulmonary Leiomyosarcoma Treated with Chemotherapy and Surgery}

Ayadi-Kaddour $A^{1 *}$, Hamrouni $\mathbf{R}^{1}$, Fekih $L^{2}$, Marghli $A^{3}$ and El Mezni $F^{1}$

${ }^{1}$ Department of Pathology, Abderrahman Mami Hospital, Ariana, Tunisia

${ }^{2}$ Department of Pulmonology, Ibn Nafis, Abderrahman Mami Hospital, Ariana, Tunisia

${ }^{3}$ Department of Thoracic Surgery, Abderrahman Mami Hospital, Ariana, Tunisia

\begin{abstract}
Primary pulmonary leiomyosarcomas are a rare malignant tumor with generally poor prognosis. They pose important problems of differential diagnosis especially with pulmonary metastatic sarcoma. They are subdivided into two categories those originating from pulmonary parenchyma and those originating from pulmonary arteries. The authors describe a case of 54-year-old woman that had her left upper lobe mass checked. The diagnosis was made based on the microscopic examination of the percutaneous trans-thoracic needle biopsy. The general examination did not show any metastatic lesions in other areas. The treatment was based on chemotherapy followed by a surgical resection. The patient did not experience recurrences or complications.
\end{abstract}

Keywords: Pulmonary sarcoma; Leiomyosarcoma; Computed tomography; Chemotherapy; Surgery

\section{Introduction}

Leiomyosarcomas are neoplasms of smooth muscles that most commonly arise from the uterus, gastrointestinal tract, or soft tissue. Primary pulmonary leiomyosarcoma is extremely rare, accounting for less than $0.5 \%$ of all malignant lung tumors [1]. They pose significant diagnostic problems and the primary site is often difficult to ascertain [2]. They are among the most frequent pulmonary sarcomas and originate from the smooth muscle of the bronchi, bronchioles or bronchial arterioles. The preoperative diagnosis is extremely difficult [1]. Because these tumors grow and metastasize slowly, they are virtually curable with early detection and treatment. The role of neoadjuvant or adjuvant chemotherapy in the treatment has not been well defined. While leiomyosarcoma tends to be resistant to chemotherapy, each case is different and results can vary widely. The authors describe an additional case of primary pulmonary leiomyosarcoma presenting as a left upper lobe mass lesion, successfully treated by chemotherapy and surgery.

\section{Case Report}

A 54-year-old-non-smoker female patient presented with a 9-month lasting dyspnea. She had noticed a weight loss of $5 \mathrm{~kg}$ over the course of the last 4 months. Physical examination was normal. Laboratory level tests were within normal values. Chest radiography showed a 13-centemeter homogenous opacity on the left side. Fiberoptic bronchoscopy showed signs of extrinsic compression of the lingular bronchus. Computed tomography scan of thorax revealed a lingular mass with heterogeneous density measuring $14.3 \times 9.4 \mathrm{~cm}$, with a fleshy peripheral component and a necrotic center compressing the lingular bronchus with no intraluminal bud (Figure 1). Computed tomographyguided transthoracic biopsy found spindle-shaped cells with atypia and mitoses compatible with a poorly differentiated sarcoma. After the pathological diagnosis, a CT scan of the whole body was performed but did not reveal any tumors. Treatment consisted in six cycles of chemotherapy based on adriamycin and cyclophosfamid followed by a regression of the tumor $(6 \times 3 \mathrm{~cm})$ with persistence of the parietal contact (Figure 2). Thereafter the patient underwent a left upper lobectomy. Macroscopic examination showed that the lobe was occupied by a large tumor of $12 \times 13 \mathrm{~cm}$, poorly limited, hard consistency and whitish in appearance, infiltrating the lobar bronchus. Microscopic examination of the resected specimen showed a highly cellular mesenchymal cell

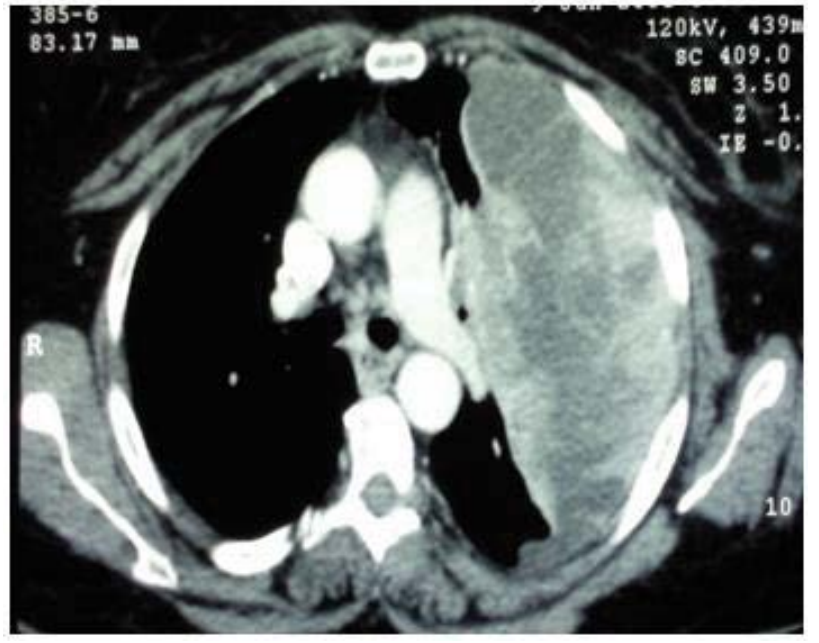

Figure 1: CT-scan: a lingular necrotic mass with heterogeneous density measuring $14 \times 9 \mathrm{~cm}$ associated with anterior mediastinal adenopathies.

proliferation made of spindle cells with eosinophilic cytoplasm often equipped with hyperchromatic and mitotic nuclei (Figures 3 and 4). The immunohistochemistry investigations showed a moderately differentiated tumor expressing smooth muscle actin, desmin and vimentin, with a negative reaction to cytokeratin and Epithelial Membrane Antigen. A diagnosis was established of primary pulmonary leiomyosarcoma. The patient made a slow but steady recovery and was discharged on the $7^{\text {th }}$ postoperative day. She has been followed up regularly and was well at the last examination, 3 years postoperatively.

*Corresponding author: Ayadi-Kaddour A, Department of Pathology, Abderrahman Mami Hospital, Ariana, Tunisia, Tel: 0021622551641; E-mail: kaddour1fr@yahoo.fr

Received March 29, 2015; Accepted April 27, 2015; Published May 04, 2015

Citation: Kaddour AA, Hamrouni R, Fekih L, Marghli A, El Mezni F (2015) Primary Pulmonary Leiomyosarcoma Treated with Chemotherapy and Surgery. J Pulm Respir Med 5: 258. doi:10.4172/2161-105X.1000258

Copyright: () 2015 Kaddour AA, et al. This is an open-access article distributed under the terms of the Creative Commons Attribution License, which permits unrestricted use, distribution, and reproduction in any medium, provided the original author and source are credited. 


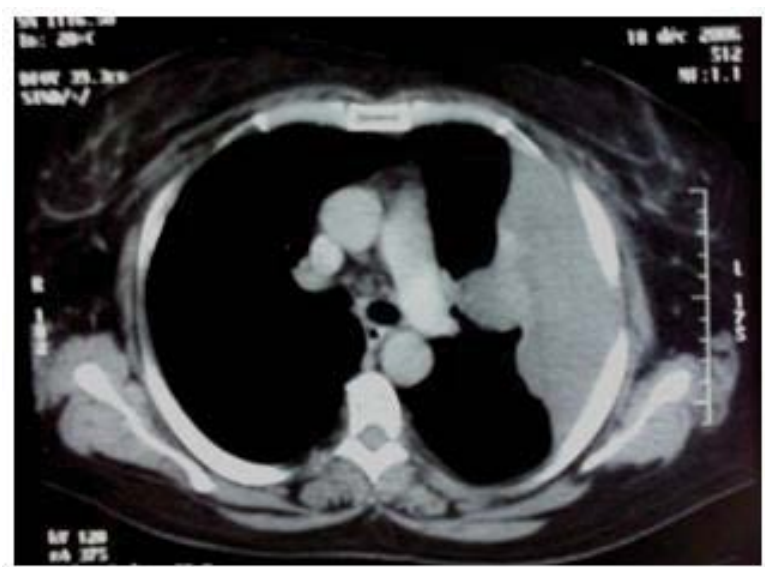

Figure 2: Post-chemotherapy CT Scan: a marked regression in tumor volume after chemotherapy $(13 \times 6 \mathrm{~cm})$

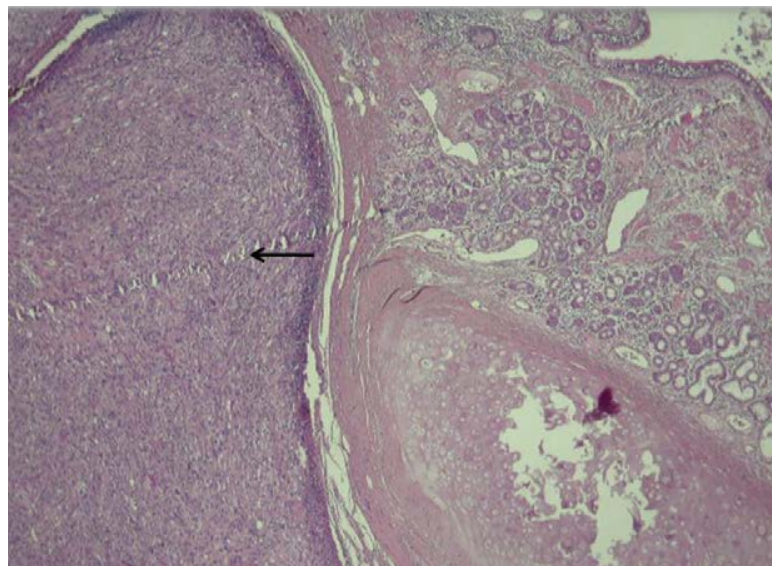

Figure 3: Pulmonary tumor composed of atypical spindle cells intermixed (HE 250x)

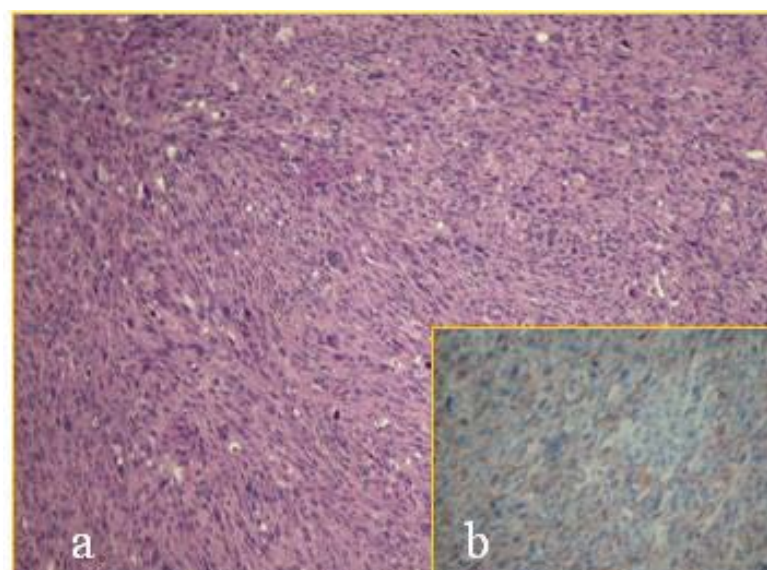

Figure 4: (a) Many atypical cells with numerous mitoses (HE 400x). (b) Immunohistochemical study showing the expression of actin muscle antigen by tumoral cells.

\section{Discussion}

Primary pulmonary sarcoma are exceptionally rare tumors that constitute only $0.2-0.5 \%$ of all lung cancers [2]. Pulmonary leiomyosarcoma is the most common pathological type. Leiomyosarcoma of the lungs, it may originate in the arteriolar walls, muscle tissue in the interstitial plane of the alveolar walls or in primitive mesenchymal cells [3]. A few cases of endobronchial localization have been described [4]. Although more often seen in adults with an average age of 60 years, it has also been described in children [1] It is more prevalent among males, with a $2.5 / 1$ male to female ratio [2]. Known risk factors include radiation therapy, chemotherapy (cyclophosphamide, melphalan, nitrosoureas), and environmental and occupational exposures (arsenic, dioxin, vinyl chloride) [5]. The clinical symptoms of these tumors are non specific and are mainly represented by dyspnea, chest pain, impaired general condition and intractable congestive heart failure if located in the pulmonary artery $[3,4]$. Primary leiomyosarcomas of the lung arise most commonly in the left lower lobe. Initially, a mass is detected on routine chest radiography. Parenchymal pulmonary leiomyosarcoma classically present on chest radiographs as a homogenous, lobulated mass with a sharply defined margins [6]. Rarely do they show cavitation to present as cystic masses. CT scan classically reveal multifocal lesions, endobronchial obstruction and the absence of hilar lymphadenopathy [4,5]. Unlike epithelial tumors, they do not exhibit any tendency towards exfoliation; hence bronchoscopy and washings or brushings are usually unrewarding [3]. Histological examination is the most reliable and confirmative method of diagnosing leiomyosarcoma and its differentiation from carcinoma. Diagnosis is made mainly based on surgical biopsy, surgical specimen or autopsy samples. The morphological features of these tumors recapitulate those seen in soft tissues. The microscopic features are a fascicular proliferation of spindle cells with scant fibrillary cytoplasm and elongated blunt-ended nuclei. The latter are monomorphic and hyperchromatic with coarse chromatin and a large number of mitotic figures [2]. Differential diagnosis arises with undifferentiated carcinoma, biphasic tumors, carcinosarcoma, carcinoid tumor, intrapulmonary thymoma and lymphoma $[7,8]$. Immunhistochemical studies help to rectify the diagnosis by showing positive reactions mostly for smooth muscle actin or desmin. The diagnosis of primary leiomyosarcoma should be entertained only when there is no evidence of an occult primary elsewhere in the body. Particularly for women the uterus should be carefully examined [1,2]. Metastasis to the lymph nodes is said to be an unusual feature of pulmonary leiomyosarcoma [6]. Distant metastasis to the liver, pleura, skin and adrenal glands have been reported [9]. Surgery is the mainstay therapy for pulmonary leiomyosarcoma if the diseases is contained and could be the best choice in some cases with minimal spread. It is to be noted that the magnitude of the resection has no bearing on the outcome, as it only reflects the size and extension of the tumor [8]. The role of radiotherapy is only palliative and chemotherapy is usually advocated for those having bilateral diseases, extra-thoracic dissemination and refusal for surgery. Patients with large primary tumors may receive preoperative radiation treatment in hopes of decreasing the size of the mass before surgical resection [7]. Depending on the size and grade of the tumor, radiation therapy, chemotherapy, or both may be recommended as an adjuvant therapy. Lymph node resections are generally not necessary because primary leiomyosarcomas of the lung, rarely show lymph node involvement. The most consistent predictor of long-term survival is the grade of the tumor. The size of the tumor, the stage at presentation and completeness of the resection are the other variables [8,9]. Prognosis is generally poor in tumors measuring more than $10 \mathrm{~cm}$ diameter and more than eight mitotic figures seen per 10 high power fields, even in 
resected cases. There seems to be a higher survival rate in patients with central than with peripheral sarcoma.

\section{Conclusion}

Primary pulmonary leiomyosarcoma is a very rare clinicalpathological disorder that requires early detection. The most consistent predictive factor for long-term survival is the tumor stage. The surgical resection should be the preferred method for definitive diagnosis and curative treatment.

\section{References}

1. Janssen JP, Mulder JJ, Wagenaar SS, Elbers HR, van den Bosch JM (1994) Primary sarcoma of the lung: a clinical study with long-term follow-up. Ann Thorac Surg 58: 1151-1155

2. Litzky LA (2008) Pulmonary sarcomatous tumors. Arch Pathol Lab Med 132: 1104-1117.
3. Shoji F, Yoshino I, Takeshita M, Sumiyoshi S, Sueishi K, et al. (2007) Pulmonary leiomyosarcoma presenting as a pancoast tumor. Pathol Res Pract 203: 745748

4. Hyung Lee S, Seung Shin J, Kyung Kim M, Ho Kang K (2001) Primary endobronchial leiomyosarcoma : diagnosis following expectoration of tumor fragment. Respiration 68: 99-102.

5. Kanematsu T, Toyoda Y, Goto H, Abe S, Kawakita N, et al. (2011) [A case of primary pulmonary leiomyosarcoma with multiple nodular lesions in the lungs]. Nihon Kokyuki Gakkai Zasshi 49: 167-171.

6. Fitoz S, Atasoy C, Kizilkaya E, Başekim C, Karsli F (2000) Radiologic findings in primary pulmonary leiomyosarcoma. J Thorac Imaging 15: 151-152.

7. Luthra M, Khan H, Suhail MF, Avadhani V (2012) Primary pulmonary leiomyosarcoma -- a case report. Arch Bronconeumol 48: 476-478.

8. Ketata W, Msaad S, Kwass H, Bahri Zouari I, Abbes K, et al. (2009) [Primary pulmonary leiomyosarcoma revealed by spontaneous pneumothorax]. Rev Pneumol Clin 65: 309-312.

9. Kosaka M, Chiaki T, Yokoyama T, Koizumi T, Shinohara N, et al. (2010) [A case of primary pulmonary leiomyosarcoma showing rapid growth and fatal outcome]. Nihon Kokyuki Gakkai Zasshi 48: 729-733. 